\title{
How Can mHealth Keep New Moms out of the Blue? Evidence of the Heterogeneous Treatment Effects on Depression from a Large-Scale Field Experiment
}

\author{
Benjamin Campbell $^{1^{*}}$; Yanfang $\mathrm{Su}^{2^{*}}$; Weiguang Wang ${ }^{3^{*}}$; Guodong $\mathrm{Gao}^{3^{*}}$ \\ ${ }^{1}$ Depression Clinical and Research Program, Massachusetts General Hospital, Boston, MA, United States \\ ${ }^{2}$ Freeman Spogli Institute for International Studies, Stanford University, Stanford, CA, United States \\ ${ }^{3}$ Smith School of Business, University of Maryland, College Park, MD, United States \\ *all authors contributed equally
}

Corresponding Author:

Benjamin Campbell

Depression Clinical and Research Program

Massachusetts General Hospital

1 Bowdoin Square

Boston, MA,

United States

Phone: 14966395

Email: bcampbell7@mgh.harvard.edu

\begin{abstract}
Background: While there is substantial evidence showing the effectiveness of mHealth in treating chronic conditions, researchers now realize that mHealth does not always benefit every patient. This heterogeneity may be due to personal factors, which moderate the effectiveness of mHealth tools. However, there is relatively little research examining these moderating factors, particularly for interventions targeting postpartum depression.

Objective: This study specifically looks at factors that could moderate the effect of an mHealth intervention on postpartum depression (PPD) among women in China, where the prevalence of PPD is 10\%-15\%. Two moderating factors of interest are the psychological factors of perceived susceptibility and intrinsic motivation. Perceived susceptibility is the expectant mother's perception of her and her baby's risk of health problems. Intrinsic motivation is the natural human propensity of an expectant mother to act for her and her baby's health. The hypotheses for this study are: 1) those with higher perceived susceptibility are more likely to benefit from the intervention, and 2) those with higher intrinsic motivation are more likely to benefit from the intervention.
\end{abstract}

Methods: One of the world's largest field experiments was conducted to explore the effectiveness of an SMS messaging application on the health of expectant mothers in China. The application delivered timely, accurate prenatal health messages, including some on postpartum depression, to pregnant women over the course of their pregnancy. The study enrolled 4,629 expectant mothers in northern China over a period of two years. Participants entered one of four groups, including three different treatment groups and a control group. Subjects were interviewed in postpartum home visits to obtain a measure of PPD. In total, PPD measures were obtained for 1,293 new mothers. Moderator variable data were reported by the expectant mothers at enrollment. In this analysis, the PPD outcome was measured between the treatment (comprising all three treatment groups) and the control group for expectant mothers with low versus high levels of susceptibility perception, and low versus high levels of intrinsic motivation.

Results: Results reveal significant moderating effects of an expectant mother's intrinsic motivation and perceived susceptibility on the effectiveness of the SMS intervention. Specifically, SMS was associated with reduced PPD score among expectant mothers with high perceived susceptibility by $31 \%$, compared to the control group. However, expectant mothers with low perceived susceptibility do not seem to benefit from the SMS messaging. Also, contrary to previous studies, results show that expectant mothers with low intrinsic motivation enjoy the benefits of the intervention by a reduction of $20 \%$ in PPD score. Furthermore, those with high intrinsic motivation showed a $17 \%$ increase in PPD score.

Conclusions: Our results suggest that if resources are limited, the most efficient way to reduce PPD in a population may be to target pregnant women with high susceptibility perception and low intrinsic motivation using SMS health messaging. Our findings 
also suggest that those with high intrinsic motivation may not be the optimal target population for clinical mobile messaging. Overall, the evidence generated from this large-scale field experiment contributes to our understanding of effective use of mHealth for postpartum depression.

(iproc 2017;3(1):e14) doi: 10.2196/iproc.8435

\section{KEYWORDS}

mHealth; postpartum depression; China

\section{Multimedia Appendix 1}

Full poster.

[PDF File (Adobe PDF File), 408KB-Multimedia Appendix 1]

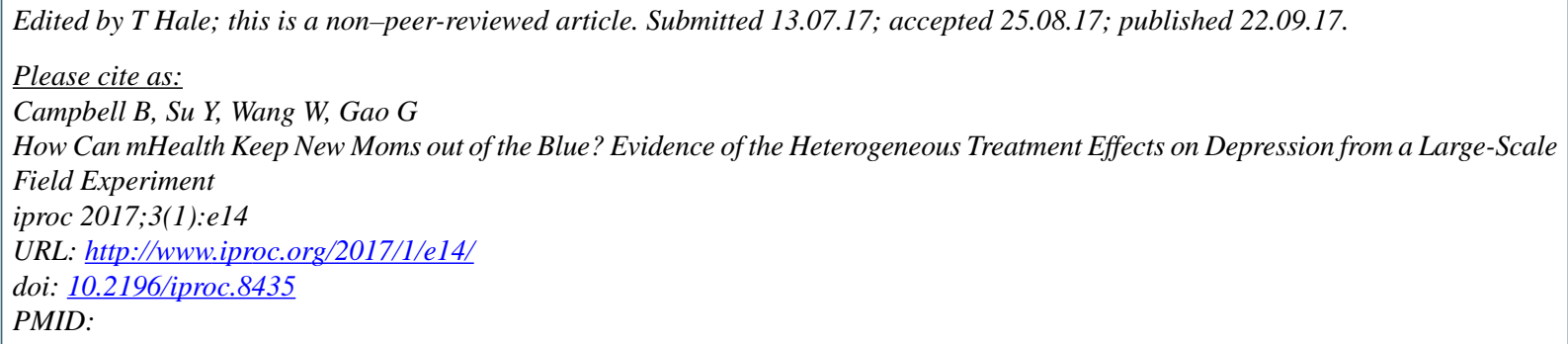

CBenjamin Campbell, Yanfang Su, Weiguang Wang, Guodong Gao. Originally published in Iproceedings (http://www.iproc.org), 22.09.2017. This is an open-access article distributed under the terms of the Creative Commons Attribution License (https://creativecommons.org/licenses/by/4.0/), which permits unrestricted use, distribution, and reproduction in any medium, provided the original work, first published in Iproceedings, is properly cited. The complete bibliographic information, a link to the original publication on http://www.iproc.org/, as well as this copyright and license information must be included. 